\title{
Statistical hadronization of charmed quarks at SPS and RHIC
}

\author{
A.P. Kostyuk ${ }^{\mathrm{a}}$ \\ ${ }^{a}$ Institut für Theoretische Physik, Universität Frankfurt, Germany \\ and \\ Bogolyubov Institute for Theoretical Physics, Kyiv, Ukraine
}

Production of open and hidden charm hadrons in heavy ion collisions is considered within the statistical coalescence model. The charmed quark-antiquark pairs are assumed to be created at the initial stage of the reaction in hard parton collisions. The number of these pairs is conserved during the evolution of the system. At the hadronization stage, the charmed (anti)quarks are distributed among open and hidden charm hadrons in accordance with the laws of statistical mechanics.

The model is in agreement with the experimental data on J/psi to Drell-Yan ratio in $\mathrm{Pb}+\mathrm{Pb}$ collisions at SPS. This agreement can be reached only if a rather strong enhancement of the open charm production in central $\mathrm{Pb}+\mathrm{Pb}$ collisions is assumed. A possible mechanism of the charm enhancement is discussed.

At the top RHIC energy, the model predicts an increase of J/psi to Drell-Yan ratio in more central nucleus-nucleus collisions with respect to less central ones.

The interest to charmed quarks in heavy ion physics was initially motivated by the suggestion of Matsui and Satz [国] to use charmonia as a probe of the state of matter created at the early stage of the collision.

The standard picture of charmonium production in nucleus-nucleus collisions assumes that charmonia are created exclusively at the initial stage of the reaction in primary nucleon-nucleon collisions. During the subsequent evolution of the system, the number of hidden charm mesons is reduced because of (a) absorption of pre-resonance charmonium states in the nuclei (normal nuclear suppression), (b) interactions of charmonia with secondary hadrons (comovers), (c) dissociation of $c \bar{c}$ bound states in the deconfined medium. It was found that the $J / \psi$ suppression with respect to Drell-Yan muon pairs measured in proton-nucleus and nucleus-nucleus collisions with light projectiles can be explained by the normal nuclear suppression alone [2]. In contrast, the NA50 experiment with a heavy projectile and target (lead-lead) revealed essentially stronger $J / \psi$ suppression for central collisions [3]. This anomalous $J / \psi$ suppression was attributed to formation of quark-gluon plasma 田.

Despite of quite successful agreement with the $J / \psi$ data, the standard scenario seems to be in trouble explaining the $\psi^{\prime}$ yield. The recent lattice simulations [5] suggest that the temperature of $\psi^{\prime}$ dissociation $T_{d}\left(\psi^{\prime}\right)$ lies far below the deconfinement point $T_{c}: T_{d}\left(\psi^{\prime}\right) \approx$ 
0.1-0.2T $[6]$. Therefore, not only the quark-gluon plasma, but also a hadronic co-mover medium should completely eliminate $\psi^{\prime}$ charmonia in central $\mathrm{Pb}+\mathrm{Pb}$ collisions at SPS. However, the experiment revealed a sizable $\psi^{\prime}$ yield (see, for instance, [7]). It was observed [8] that $\psi^{\prime}$ to $J / \psi$ ratio decreases with centrality only in peripheral lead-lead collisions, but remains approximately constant at sufficiently large number of participants $N_{p} \geq 100$.

A completely different picture of charmonium production was proposed in Ref. [9]: hidden charm mesons are supposed to be created at the hadronization stage. Similar to all other hadrons, their abundancies can be described within the thermal model [10]. However, production of heavy quarks in soft processes is expected to be negligible. Most likely, they are produced exclusively at the hard stage. Their number, therefore, can, generally speaking, deviate from the thermal equilibrium value. This fact was taken into account by the statistical coalescence model (SCM), which was proposed in Ref.[11]. Soon, it was realised that, when the average number of heavy quark pairs per collision is small $(\lesssim 1)$, exact conservation of this pairs in the hadronization process appears to be crucial $[12]$.

In this talk, I present the results [13,14 obtained within the statistical coalescence model:

- $c$ and $\bar{c}$ are created at the initial stage of the reaction in primary hard parton collisions;

- their number remains approximately unchanged during the subsequent evolution;

- they are distributed over open charm hadrons and charmonia at the hadronization stage in accordance with laws of statistical mechanics.

Within its applicability domain $\left(N_{p} \gtrsim 100\right)$, the model demonstrates excellent agreement $\left(\chi^{2} /\right.$ dof $\left.=1.06\right)$ with the NA50 data on $J / \psi$ production (see Fig. 1).

The so-called 'second threshold of anomalous $J / \psi$ suppression' at large transverse energy $\left(E_{T} \gtrsim 100 \mathrm{GeV}\right)$ naturally appears in SCM due to two effects: (i) fluctuations of the transverse energy at fixed number of participant nucleons and (ii) $E_{T^{-}}$-losses in the dimuon event sample with respect to the minimum bias one (see Ref. [14] for details).

The agreement with the data is reached assuming a rather strong enhancement of the open charm production in central $\mathrm{Pb}+\mathrm{Pb}$ collisions: up to a factor of about 3.5 within the rapidity window of the NA50 spectrometer. This is consistent with the indirect experimental result [15].

A possible mechanism of open charm enhancement was proposed in Ref. [16]. Calculations in the leading order of perturbative quantum chromodynamics (QCD) show that, assuming factorization (i.e. independence of the hard QCD process on the subsequent soft stage), a great fraction of $c \bar{c}$ pairs is created with invariant masses $M_{c \bar{c}}$ below the corresponding meson threshold $2 m_{D}$. In vacuum, these subthreshold pairs would not be able to form observable hadrons. In reality, however, some part of these pairs may hadronize due to interaction with spectator partons. Nevertheless, there is no reason to assert that all subthreshold pairs result in final state hadrons with open or hidden charm. It is quite reasonable to assume that a great part of them has to annihilate into lighter hadrons, or, alternatively, the final state interactions prevent formation of such pairs (factorization is broken). 


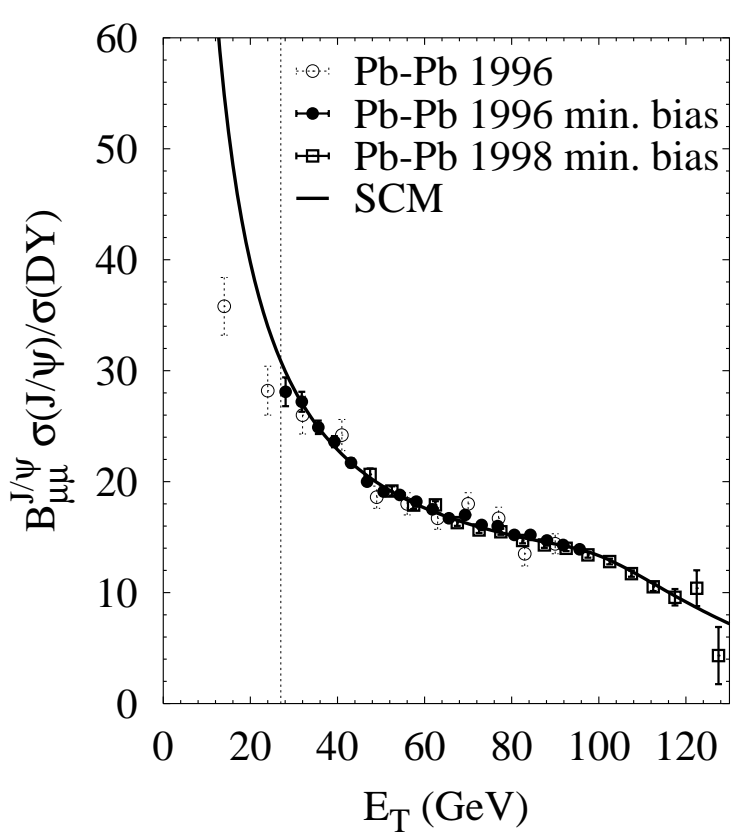

Figure 1. The dependence of the $J / \psi$ to Drell-Yan ratio on the transverse energy at SPS. The vertical line shows the boundary of the applicability domain of the statistical coalescence model (SCM), see [14] for details.

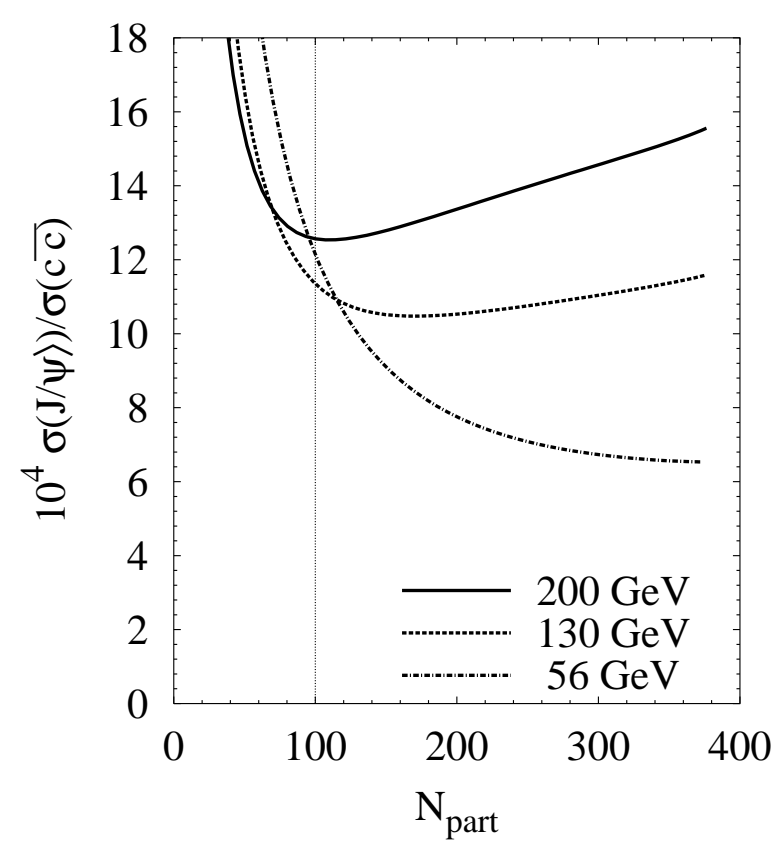

Figure 2. The dependence of the $J / \psi$ to open charm ratio on the number of nucleon participants at RHIC. The vertical line shows the boundary of the applicability domain of the statistical coalescence model (SCM).

In presence of a deconfined medium (quark-gluon plasma or its precursor), the subthreshold quark pairs can hadronize due to interactions with the medium, or, alternatively, the medium can modify the final state interactions making possible creation of the subthreshold pairs and their subsequent hadronization. This should lead to enhanced production of the total charm in nucleus-nucleus collisions in comparison to the standard result obtained within the direct extrapolation of nucleon-nucleon data.

We have estimated the upper bound of the open charm enhancement. At SPS energies, one can expect maximal enhancement by a factor of about 6 , which is consistent with our above fit for the $J / \psi$ to Drell-Yan ratio.

It is interesting to note, that the statistical coalescence model does not always lead to $J / \psi$ suppression. Predictions of the statistical coalescence model for RHIC are shown in Fig. 1 (see also Ref.[17]). As is seen, $J / \psi$ suppression is still expected at the lowest RHIC energy. In contrast, relative $J / \psi$ enhancement (e.g. growth of the $J / \psi$ to open charm円 ratio with the number of participants at $\left.N_{p}>100\right)$ is predicted for the top RHIC energy.

In conclusion, the NA50 data for not very peripheral lead-lead collisions are consistent with the following scenario:

\footnotetext{
${ }^{1}$ It should be stressed that, due to possible nuclear shadowing, the open charm multiplicity has not to be proportional to the number of binary nucleon-nucleon collisions. Therefore the ratio of $J / \psi$ multiplicity to the number of binary collisions may decrease even if $J / \psi$ to open charm ratio increases.
} 
Formation of deconfined medium leads to enhanced production of charmed hadrons. Charmonia as well as other hadrons are formed at the hadronization stage. The distribution of charm quarks and antiquarks over open and hidden charm hadrons follows laws of statistical mechanics.

A relative $J / \psi$ enhancement is expected at the top RHIC energy.

I am indebted to M. Gorenstein, W. Greiner, L. McLerran and H. Stöcker for fruitful collaboration. I wish to thank A. Andronic, F. Becattini, P. Bordalo, P. BraunMunzinger, L. Bravina, A. Capella, Yu. Dokshitzer, J. Cleymans, W. Florkowski, A. Frawley, L. Grandchamp, A. Kaidalov, B. Kämpfer, J. Kapusta, F. Karsch, D. Kharzeev, V. Koch, K. Redlich, D. Rischke, M. Rosati, H. Satz, E. Shuryak, Yu. Sinyukov, J. Stachel, J. Wambach, A. Zhitnitsky and G. Zinovjev for interesting discussions.

\section{REFERENCES}

1. T. Matsui and H. Satz, Phys. Lett. B 178 (1986) 416;

H. Satz, Rept. Prog. Phys. 63 (2000) 1511.

2. M. C. Abreu et al., Phys. Lett. B 466 (1999) 408.

3. M. C. Abreu et al. [NA50 Collaboration], Phys. Lett. B 410 (1997) 337;

M. C. Abreu et al. [NA50 Collaboration], Phys. Lett. B 450 (1999) 456.

4. M. C. Abreu et al. [NA50 Collaboration], Phys. Lett. B 477 (2000) 28.

5. F. Karsch, E. Laermann and A. Peikert, Nucl. Phys. B 605 (2001) 579.

6. S. Digal, P. Petreczky and H. Satz, Phys. Lett. B 514 (2001) 57.

7. M. C. Abreu et al. [NA50 Collaboration], LIP-96-04 Invited talk at 26th International Symposium on Multiparticle Dynamics (ISMD 96), Faro, Portugal, 1-5 Sep 1996.

8. H. Sorge, E. Shuryak and I. Zahed, Phys. Rev. Lett. 79 (1997) 2775.

9. M. Gazdzicki and M. I. Gorenstein, Phys. Rev. Lett. 83 (1999) 4009.

10. P. Braun-Munzinger, I. Heppe and J. Stachel, Phys. Lett. B 465 (1999) 15;

F. Becattini, J. Cleymans, A. Keranen, E. Suhonen and K. Redlich, Phys. Rev. C 64 (2001) 024901;

G. D. Yen and M. I. Gorenstein, Phys. Rev. C 59 (1999) 2788.

11. P. Braun-Munzinger and J. Stachel, Phys. Lett. B 490 (2000) 196.

12. M. I. Gorenstein, A. P. Kostyuk, H. Stöcker and W. Greiner, Phys. Lett. B 509 (2001) 277; J. Phys. G 27 (2001) L47.

13. A. P. Kostyuk, M. I. Gorenstein, H. Stöcker and W. Greiner, Phys. Lett. B 531 (2002) 195.

14. A. P. Kostyuk, M. I. Gorenstein, H. Stöcker and W. Greiner, J. Phys. G 28 (2002) 2297.

15. M. C. Abreu et al. (NA38 and NA50 Collaborations) 2000 Eur. Phys. J. C 14443.

16. A. P. Kostyuk, M. I. Gorenstein and W. Greiner, Phys. Lett. B 519 (2001) 207.

17. M. I. Gorenstein, A. P. Kostyuk, L. McLerran, H. Stöcker and W. Greiner, J. Phys. G 28 (2002) 2151;

M. I. Gorenstein, A. P. Kostyuk, H. Stöcker and W. Greiner, Phys. Lett. B 524 (2002) 265. 